\title{
Analysis and Improvement of Identification Method for Critical Unit in Transient Process of Multi-generator Power System
}

\author{
Wenyong $\mathrm{Li}^{1,}$, , Hongsheng $\mathrm{Su}^{1, \mathrm{~b}}$ \\ ${ }^{1} 028$ mailbox, Lanzhou Jiaotong University, Lanzhou 730070, Gansu Province, China \\ a12569528@qq.com, ${ }^{\mathrm{b}}$ shsen@163.com
}

\begin{abstract}
Keywords: Extended Equal Area Criterion (EEAC); multi-generator power system; Power System Analysis Software Package(PSASP); transient stability; critical unit identification; unit power difference

Abstract. The extended equal area criterion (EEAC) is one of the most important methods to identification the stability of Multi-generator Power System, and the key of this method is to correctly identify the critical unit. However, critical units of the existing recognition methods exist some problems, such as the existing methods in the early fault can not correctly identify critical units, and need to adopt different parameters to identify critical units in different situations. Based on the above problems, this paper analyzes the advantages and disadvantages of the existing critical unit identification methods, and proposes an improved method for the identification of critical units. The improved critical unit identification method uses the real time difference between the mechanical power and the electromagnetic power of the generator, and the difference is defined as the unit power difference. Finally, the simulation and analysis results show that the improved method can identify the critical unit more quickly and accurately.
\end{abstract}

\section{Introduction}

The interconnection between regional power grids is becoming more and more widespread with power system development, which not only brings great benefits to the social and economic development, but also generates serious security risks to huge power grid with complicated structure due to transient stability problems. Several classical cases illustrated that some events with very low probability might also cause power grid splitting or even large-scale blackout accident for the interconnected power grid with a strong structure, for example, the large-scale blackout of power grid in the United States, and Canada, and as well as India[1-3]. Hence, the higher requirements expected to be put forward for the emergency control of transient stability of interconnected power network [4].

Since power system is a high-dimensional nonlinear dynamic system, the stability of which can be solved using the numerical method alone but not be expressed by mathematical analysis. Usually, this is very time-consuming and not conducive to emergency control of the system. Hence, the current widely used stability control system conducts transient stability control trough online queries countermeasure table generated offline after disturbance or failure [5-7]. This method possesses two flaws: one is that countermeasure table is impossible to consider all the circumstances, and if there are no corresponding countermeasures, the control system can only handle the relatively severe cases, which will lead to excessive control. The other is that the numerical calculation is particularly large for large systems, and the system structure is constantly changing, and recalculating is required whenever the system structure changes [8-9].

The control method to refresh decision table online is a current hot research topic. In [10], the idea of online pre-decision and real-time matching is proposed for the first time, and a set of online pre-decision control system is developed using the parallel computing and Extended Equal Area Criterion (EEAC), which makes the emergency control technology move a big step forward [11]. However, the critical unit identification, a key step of online decision-making, may be misjudged in the stable boundary, which will cause emergency control failure, and then the system to lost instability can only be resolved by splitting of the last defense line of power system, which may cause large-scale blackout of the regional power grid [12]. Therefore, to identify critical unit is a key step to perform system stability identification and real-time emergency control using EEAC criterion. 
Presently, there are two main ways to identify the critical unit. One is to use the real-time power angle data of the generator[13], and the problem is that the correct rate of critical unit identification is not high in the early stage of fault. The other is to use the real-time power angle and angular velocity variation of the generator, and the problem is that different situations need to choose the corresponding parameters correctly[14]. In this paper, a new method of critical unit identification is proposed to improve the above problems, and the advantages of this method are discussed by simulation and comparison.

\section{Critical unit group identification method in EEAC}

EEAC criterion is to apply the gaps between the generator power angle $\delta$ to discriminate the critical unit group. The specific method is that after the system receives the impact, the collected power angles of the generator are arranged from small to large, and the generator is divided into the critical unit S-group and the non-critical unit A-group from the place with the largest gap between front and rear in this arrangement.

$$
\left.\begin{array}{l}
\frac{\mathrm{d} \theta}{\mathrm{dt}}=\Delta \omega \omega_{0} \\
\frac{\mathrm{d} \omega}{\mathrm{dt}}=\frac{1}{T_{j}}\left(P_{m}-P_{0}\right)
\end{array}\right\}
$$

Equation (1) is the motion equation of generator rotor, from which it can be seen that the changes of the generator power angle and angular velocity relate to not only the input mechanical power $P_{m}$ and output electromagnetic power $P_{e}$ of the generator, but also the tectonic $T_{j}$ rotational inertia of the generator. In a short period of the system failure or disturbance, there is no obvious angle difference for the power angle of the generator unit due to the large inertia of the generator rotor. From this analysis, it can be concluded that the critical unit identification method of EEAC can not correctly identify the critical unit in some cases.

\section{Identification method of critical unit group based on the composite power angle}

To overcome the flaws in section 3, a method to determine the critical unit group by means of the composite power angle proposed can be described by in [14]

$$
\delta_{c o m}=\delta+k \Delta \omega
$$

where $\delta$ is the relative power angle of the generator, $\Delta \omega$ is the variable quantity of angular velocity, and $k$ is the setting value. The decision method is to arrange $\delta_{\text {oom }}$ from small to large, and the unit group is divided into critical unit and non-critical unit at the place with the largest gap.

Compared with the identification method of critical unit in EEAC, the advantage of this method is

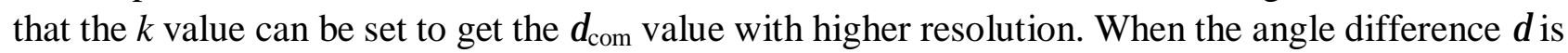
small, the units are grouped by the variation of the power angle and the angular velocity. However, its disadvantage is the need to collect two variable power angles and angular velocities. And in different circumstances, in order to get the ideal discrimination of the composite power angle of the generator, the $k$ value will be different. How to select the appropriate $k$ value is also a difficult thing. 


\section{Identification method of critical unit group based on unit power difference of generator}

Because there are some shortcomings for the above methods in the critical unit identification, this paper proposes a critical unit identification method based on the unit power difference of the generator. For single-machine system, the generator rotor equation during fault can be expressed [15-16]

$$
\left.\begin{array}{l}
\frac{d \delta}{d t}=\Delta \omega \omega_{0} \\
\frac{d \omega}{d t}=\frac{1}{\pi_{j}}\left(P_{m}-P_{\theta}\right)
\end{array}\right\}
$$

In (3), $\boldsymbol{P}_{m}$ is the generator's mechanical power, and $\boldsymbol{P}_{\boldsymbol{q}}$ is the generator's electromagnetic power. In the method of discriminating the composite power angle, $\Delta \omega$ can be regarded as the change rate of the power angle $\delta$ in the equation (3). By taking the derivative on both sides of the first formula in (3), it can be obtained as:

$$
\frac{d}{d t}\left(\frac{d \delta}{d t}\right)=\frac{\Delta \omega \omega_{0}}{d t}=\omega_{0} \frac{d \omega}{d t}
$$

this formula is substituted into the second formula of (3), then:

$$
\frac{d}{d t}\left(\frac{d \delta}{d t}\right)=\frac{\omega_{g}}{\tau_{j}}\left(P_{m}-P_{g}\right)=\Delta P_{m-g}
$$

In (5), $\frac{\omega_{Q}}{T_{j}}\left(P_{m}-P_{\theta}\right)$ is defined as the unit power difference $\Delta P_{m-g}, \omega_{0} / T_{j}$ is the constant associated with the generator itself. When the unit power difference $\Delta P_{m-q}$ is equal to 0 during the steady-state operation, the power angle of the generator will not change; when the unit power difference $\Delta P_{m-q}$ is greater than 0 , the input mechanical power of the generator will be greater than the output electromagnetic power, and the acceleration power angle of the generator rotor will increase; when the unit power difference $\Delta P_{m-g}$ is less than 0 , the input mechanical power of the generator will be less than the output electromagnetic power, and the deceleration power angle of the generator rotor will decrease.

In the case of stable unit power difference, the acceleration or deceleration speed of the generator rotor is related to the rotor inertia of the generator. In the transient process, the input mechanical power must be larger than the output electromagnetic power for the critical unit group; and also in the case of the generator rotor acceleration, $\Delta \boldsymbol{P}_{m-\infty}$ of the unit with the first instability is larger than that of other units, so the unit power difference value $\Delta P_{m-\varepsilon}$ can be used to identify the critical unit group.

\section{Simulation and comparison}

The three kinds of critical unit identification methods are simulated and analyzed as follows. The simulation model is an 8 -machine 36 -node model of $500 \mathrm{kV}-220 \mathrm{kV}$ power grid in a region, as shown 
in Fig.1, and the simulation platform is a comprehensive analysis program of the PSASP power system. The simulation fault is a three-phase short circuit of AC25 AC line.

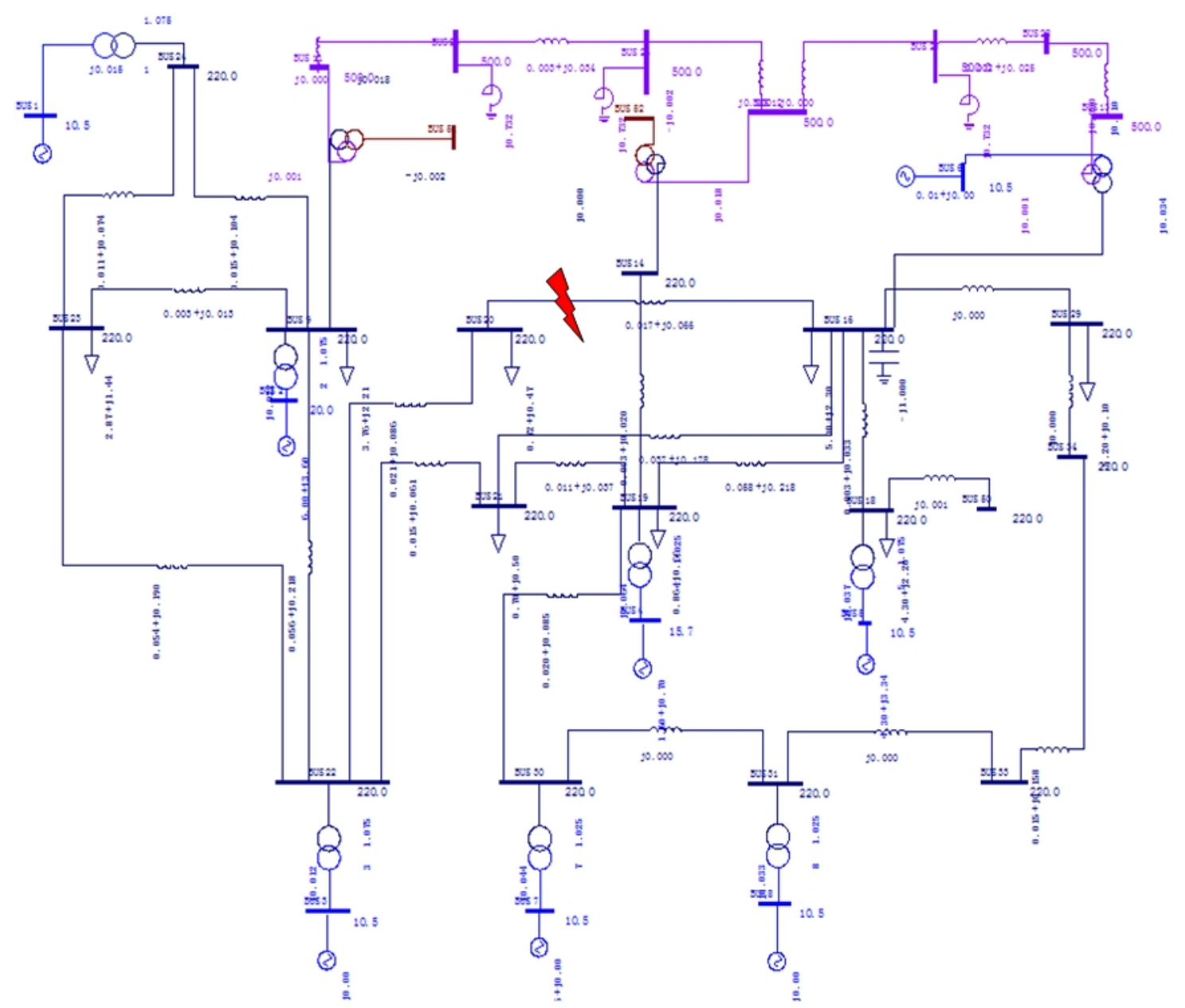

Fig.1 8-machine 36-node power grid model

Simulation of critical unit identification in EEAC. The data in Table 1 are the relative power angles at different times for the generators G1-G8 after the fault is removed.

Table1 Data sheet of relative power angle for generators

\begin{tabular}{|c|c|c|c|c|c|c|c|c|}
\hline \multirow{2}{*}{$\begin{array}{c}\text { Time } \\
\text { t/s }\end{array}$} & \multicolumn{7}{|c|}{ Gelative power angle of the generator/deg } \\
\cline { 2 - 10 } & G1 & G2 & G3 & G4 & G5 & G6 & G7 & G8 \\
\hline 0.07 & 0.063 & 0.402 & 0.076 & 0.332 & 0.224 & 0.042 & 0.253 & 0.247 \\
\hline 0.12 & 0.381 & 2.472 & 0.469 & 2.083 & 1.418 & 0.233 & 1.562 & 1.500 \\
\hline 0.15 & 1.630 & 9.535 & 2.008 & 8.377 & 5.927 & 0.898 & 6.256 & 5.926 \\
\hline 0.25 & 7.768 & 33.052 & 9.170 & 32.34 & 25.546 & 3.599 & 25.044 & 23.664 \\
\hline
\end{tabular}

It can be seen from the analysis of Table 1 that the generators' relative power angles are arranged from small to large as follows at $0.12 \mathrm{~S}$ after the fault occurs:

$\delta 6<\delta 1<\delta 3<\delta 5<\delta 8<\delta 7<\delta 4<\delta 2$ 
The maximum gap is between $\delta 3$ and $\delta 5$, and the gap is $0.949^{\circ}$. Therefore, G2, G4, G5, G7 and G8 can be identified as the critical unit group, G1, G3 and G6 are the non-critical unit group, and their relative power angle curve is as shown in Fig. 2.

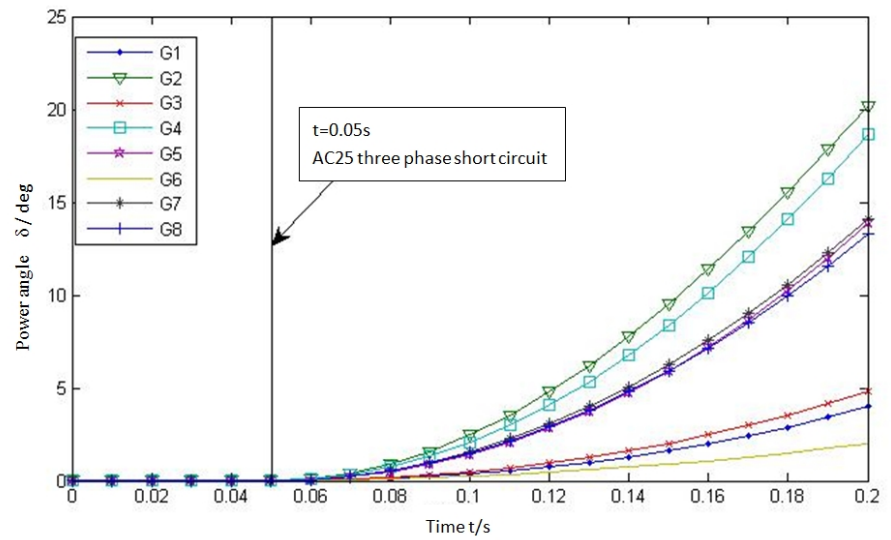

Fig.2 Relative power angle curve of generator during three-phase short circuit

It can be seen from Table 1 and Fig. 2 that the gap of the relative power angle has obvious difference after $0.1 \mathrm{~s}$ of fault, and the value is not big, and there is almost no difference in the initial time of the fault.

Simulation of critical unit identification for composite power angle. In the simulation of critical unit identification for composite power angle, $k=0.01, k=0.1$ and $k=1$ are taken respectively for comparison. It can be seen from Fig. 3 that when $k=0.01$, there is no obvious difference from using the power angle discriminant curve of the generator; when $k=0.1$ and $k=1$, the performance of using the maximum gap discrimination of composite power angle is better, and when $k=1$, it is more obvious in the initial time, that is to say, discrimination can be made earlier.

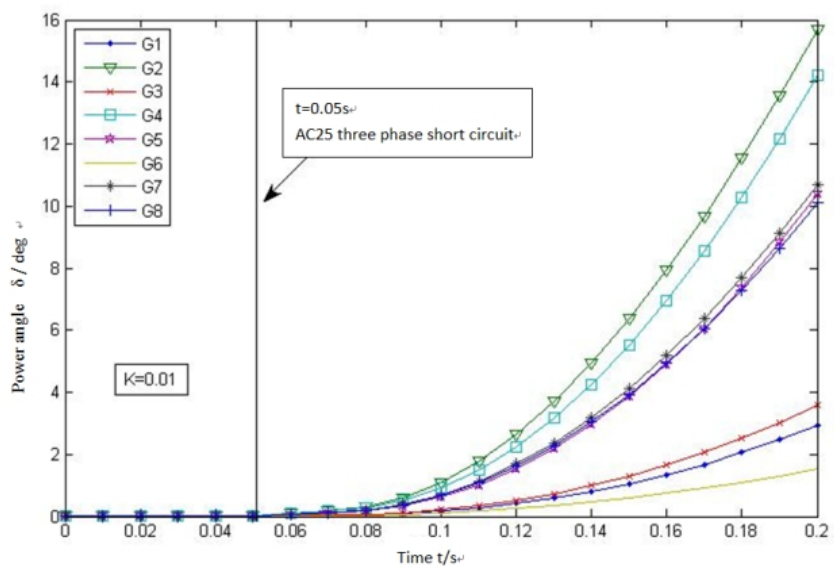

i). Composite power angle curve $(k=0.01)$ 


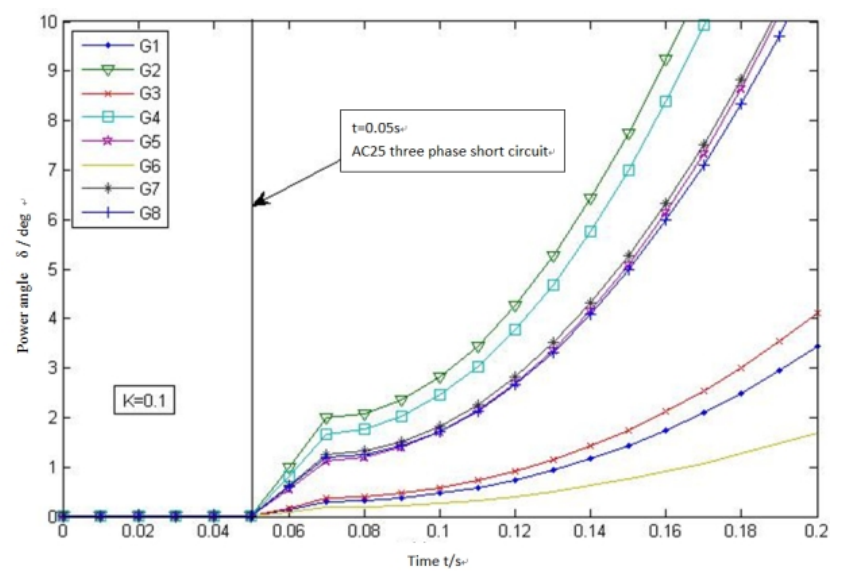

ii). Composite power angle curve $(k=0,1)$

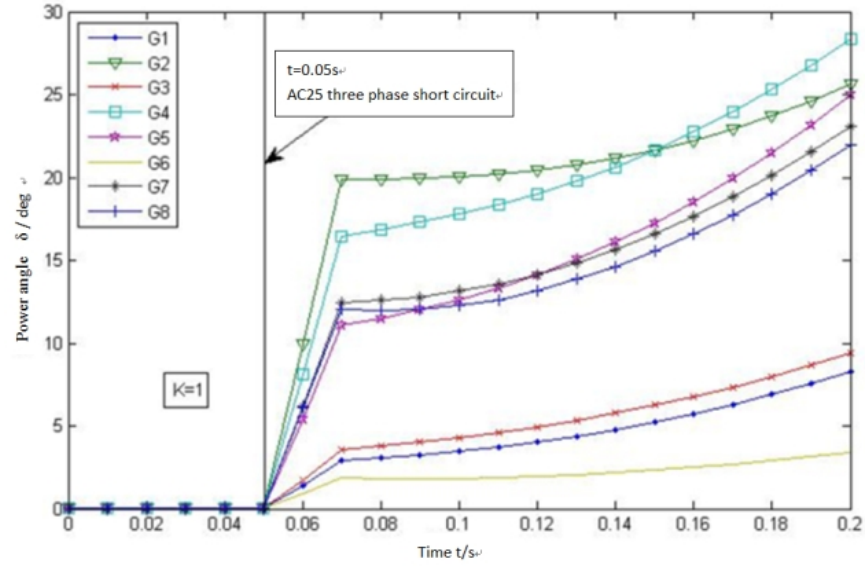

iii). Composite power angle curve $(\mathrm{k}=0.01)$

Fig.3 Composite power angle curve with different values of $\mathrm{K}$

It can be seen from Fig. 3 that the critical unit group in the transient process can be discriminated by the composite power angle, which not only better conform to the power angle trend, but also has better discriminating degree in the case that $k$ value is more reasonable. As the reasonable $k$ values are different in the different cases, it is not easy to find a reasonable value in the case of complex fault.

Simulation of identification method for unit power difference critical unit. It can be seen from Table 2 and Figure 4 that the unit power difference $\Delta \mathrm{Pm}$-e of each unit has changed obviously during the initial $0.01 \mathrm{~s}$ time in the transient process, and the gap between the units G3 and G5 is the largest, and the order of magnitude is in $10^{2}$, and the change trend of $\Delta \mathrm{Pm}-\mathrm{e}$ and the result of discrimination are the same as those of power angle discrimination and complex power angle discrimination, and the discrimination can be completed within $0.01 \mathrm{~s}$ after the fault occurs, and it does not need to carry out parameter selection when the complex power angle is discriminated.

Table $2 \Delta P_{m-e}$ value table of the initial period for fault of each generator unit

\begin{tabular}{|c|c|c|c|c|c|c|c|c|}
\hline \multirow{2}{*}{$\begin{array}{c}\text { Time } \\
\mathrm{t} / \mathrm{s}\end{array}$} & \multicolumn{8}{|c|}{ Generator relative power angle/deg } \\
\hline & G1 & 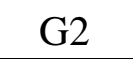 & G3 & G4 & G5 & G6 & G7 & G8 \\
\hline 0. & 291.4 & 2 & 57.7 & 644 & 1104.4 & 184.6 & 12 & 1205. \\
\hline 0.02 & 303.0 & 19 & 376.7 & 1677 & 114 & 180.2 & 12 & 1186 \\
\hline 0.03 & 316.3 & 1954.3 & 394.9 & 1697.2 & 1178.2 & 176.7 & 1253.9 & 1175. \\
\hline
\end{tabular}




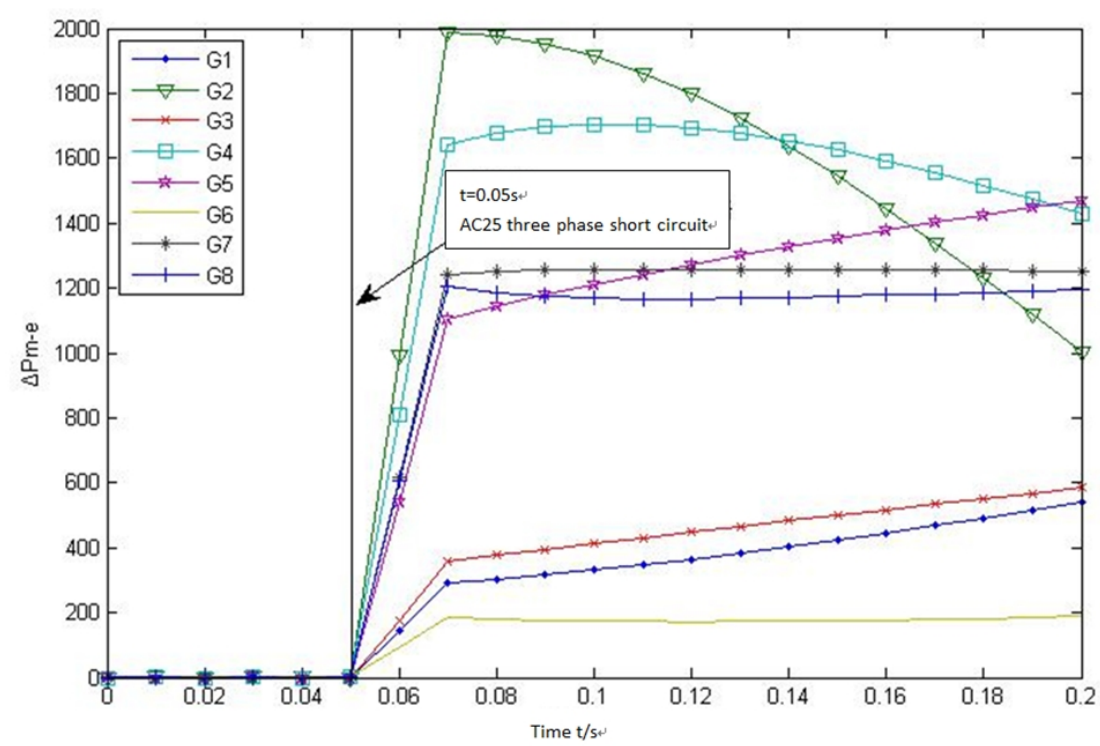

Fig.4 Curve of $\triangle P m-e$ varying with the time in the transient process for the multi-generator system

\section{Conclusion}

It can be seen from the above analysis that using the unit power difference $\Delta P m-e$ can effectively and quickly identify the critical unit group in the transient process. Although the data within very short time in the initial period of fault can be used for accurate identification, it needs to capture the generator power angle and make derivative operation, which virtually increasing the entire time of identification process. With the increasing operational speed of computer, we believe that the process of data calculation will be greatly reduced, thus ultimately providing the basis for the practical application of this method.

\section{References}

[1] Yin Yonghua, Guo Jianbo, Zhao Jianjun, et al. Preliminary Analysis and Lessons on the "8.14" Blackout in the United States and Canada [J]. Power System Technology, 2003, 27 (10): 1-5.

[2] Ma Jing, Gao Xiang, Li Yinan, et al. Analysis of Power Angle Stability for Multi-Mode Power Systems with Cascading Failures [J]. Proceedings of the CSEE, 2016, 36 (14).

[3] Xue Yusheng, Wu Yongjun, Xie Yunyun, et al. Stability Analysis of Power System under Complex Natural Disasters [J]. Automation of Electric Power Systems, 2016 (4): 10-18.

[4] Li Bijun, Hou Yuqiang. Study of Emergency Load Regulation for Security and Stability Emergency Control [J]. Power System Protection and Control, 2016, 44 (11).

[5] Ni Yixin. Theory and Analysis of Dynamic Power System [M]. Tsinghua University Press, 2002.

[6] Lu Fang, Yu Jilai. A Fast Method for Transient Stability Assessment Based on Wide-Area Phasor Measurement [J]. Automation of Electric Power Systems, 2010, 34 (8): 24-28.

[7] Zhang Baohui, Yang Songhao, Wang Huaiyuan, et al. Closed-loop Control of Transient Stability in Power Systems (2) - Method for Transient Instability Detection of Multi-machine Power Systems [J]. Automation of Electric Equipment, 2014, 34 (9): 1-6.

[8] Qin Xiaohui, Bi Tianshu, Yang Qixun, et al. Power-angle Instability Criterion Based on WAMS Dynamic Trajectory [J]. Automation of Electric Power Systems, 2008, 32 (23): 18-22.

[9] Wang Xiaoming, Liu Dichen, Wu Jun, et al. Analysis of Power System Transient Stability Based on Energy Function Method [J]. Power System Technology, 2011, 35 (8): 114-118. 
[10] Xue Y. An Emergency Control Framework for Transient Security of Large Power Systems [C]. International Symposium on Power Systems, Singapore, 1993, 120-125.

[11] Fang Yongjie, Dai Yongrong, Li Lei, et al. The OPS-1 On-line Pre-decision Based Transient Control System [J]. Automation of Electric Power Systems, 2000, 24 (3): 56-59.

[12] Yuan Jixiu. Emergency Control for Preventing Widespread Blackout of Power System-The Third Line of Defense for Safe and Stable Operation of Power System [J]. Power System Technology. 1999, 23 (4): 1-4.

[13] Wang Yang Zi, Chen Yanbo, Ma Jin. A criterion for fast stability of power system based on extended equal area criterion [J]. Science and Technology and Engineering, 2014, 14 (33): 224-228

[14] Teng Lin, Liu Wanshun, Yun Zhihao, et al. Study on Real-time Emergency Control of Power System Transient Stability [J]. Proceedings of the CSEE, 2003, 23 (1): 64-69.

[15] Li Guangqi. Transient Analysis of Power System [M]. China Electric Power Press, 2007.

[16] Li Zhiqiang, Tang Yong, He Fengjun, et al. Method for Parameter Identification of Synchronous Generator Based on Time-Frequency Transformation [J]. Proceedings of the CSEE, 2014 (19): 3202-3209. 